\title{
CT-Guided Core Needle Biopsy of Peripheral Lung Lesions with Onsite Adequate Evaluation: Review of 215 Cases
}

\author{
Li Liang, Jing Liu, Jamie Buryanek and Songlin Zhang*
}

Department of Pathology and Laboratory Medicine, The University of Texas Health Science Center at Houston, Texas, USA

\begin{abstract}
Background: CT-guided core biopsy and fine needle aspiration have been used for diagnosis of peripheral lung lesions, and both methods have advantages and disadvantages. In our institution, CT-guided core needle biopsy with onsite cytology adequacy evaluation of touch preparations is the standard method for evaluation of peripheral lung lesions.

Methods: The study was approved by Institutional Review Board. We retrospectively reviewed the cytology reports of all lung core needle biopsies during 2009-2012. The clinical information was collected from chart review.

Results: There were total 215 cases during the study period. The average lesion size (in 190 available cases) was $3.46 \mathrm{~cm}$. The average passes were 3.3/lesion. The final diagnoses included 132 malignancies, 70 benign and 13 atypical. Twenty-four cases had follow-up resections. There were two false negative diagnoses due to sample errors and there was no false positive case with a positive predictive value of $100 \%$. The final adequate rate was $99.5 \%$ (214/215), and only one case was unsatisfactory. In the 204 patients with available information of post-procedure chest X-ray, $140(68.6 \%)$ had no pneumothorax, and $64(31.4 \%)$ had pneumothorax including 48 mild $(23.5 \%), 3$ moderate $(1.5 \%), 2$ severe (1\%), 3 tension pneumothorax $(1.5 \%)$, and 8 with chest tubes placed during procedure $(3.9 \%)$.

Conclusions: Our study showed that CT-guided core biopsy with onsite cytology evaluation has high adequate and low atypical rates. Lung core biopsy can provide more accurate tumor classification and specific diagnoses for non-neoplastic lesions. Adequate tissue is often available for molecular study. The complication rate of pneumothorax in core needle biopsy is compatible to that of fine needle aspiration.
\end{abstract}

Keywords: Computed tomography; Core needle biopsy; Rapid onsite evaluation; Lung cancer

\section{Introduction}

Lung cancer is the leading cause of cancer-related death in the United States for both males and females. According to United States Cancer Statistics (USCS), in 2009, 205,974 people in the United States were diagnosed with lung cancer, including 110,190 men and 95,784 women and 158,081 people died from lung cancer, including 87,694 men and 70,387 women [1]. Lung is also one of the most common metastatic sites of many other primary cancers. Tissue is often required to differentiate primary from metastasis, benign lesions from malignant lesions, and further classify a primary lung cancer for treatment. Tissue can be either cytology or histology specimens. The cytology specimens include sputum, bronchial brushing, washing, alveolar lavage, and fine needle aspiration (FNA). FNA includes CT guided (CT-FNA), Wang needle and endobronchial ultrasound guided (EBUS). CT-FNA is often used for peripheral lung lesions and CT-guided core needle (CT-CN) biopsy can also be used for that purpose. Both CT-FNA and CT-CN have been documented to be effective for malignancy diagnosis. The most common complication is pneumothorax, and hemorrhage while air embolism and tumors seeding are rare in both fine needle aspiration and core needle biopsies [2-7].

CT-CN may have advantage over CT-FNA for obtaining more tissue to perform immunohistochemistry and/or molecular studies $[2,3]$. A specific lung cancer classification is often mandatory using the limited tissue material, because of the availability of variable targeted treatments. In addition, some treatment may improve outcome in a patient subpopulation, but contraindicated in patients with other type of tumor. For example, Bevacizumab, a monoclonal antibody against vascular endothelial growth factor (VEGF) can only be used in patients with non-squamous cell lung carcinoma, due to fatal hemorrhagic events in patients with squamous cell lung carcinoma [8]. With the demands of personalized medicine and improved understanding of the molecular pathways of different lung cancers, multiple analyses are needed, such as EGFR and KRAS mutation analysis and $A L K$ translocation $[9,10]$. In 2011, a multidisciplinary expert panel representing the International Association for the Study of Lung Cancer (IASLC), the American Thoracic Society (ATS), and the European Respiratory Society (ERS) proposed a major revision of lung cancer classification, which also emphasizes that the tissue sample should be preserved for not only morphological diagnosis, but also molecular testing [11]. Furthermore, CT-CN may provide more specific diagnosis for non-neoplastic lesions than CT-FNA.

Touch preparation from CT-CN with onsite rapid cytology evaluation can provide adequate assessment and triage specimens for appropriate studies such as flow cytometry or microbiology culture. The onsite cytology evaluation also can reduce unnecessary passes, improve the diagnostic rate and provide a preliminary cytologic diagnosis. In our institution, CT-CN with onsite cytology adequate evaluation is the standard method for evaluation of peripheral lung lesions, and here we report our institutional experience.

*Corresponding author: Songlin Zhang, Department of Pathology and Laboratory Medicine, UT Health Science Center at Houston, 6431 Fannin Street, Houston, TX 77030, USA, Tel: 713-500-5321; E-mail: songlin.zhang@uth.tmc.edu

Received September 26, 2014; Accepted November 14, 2014; Published November 16, 2014

Citation: Liang L, Liu J, Buryanek J, Zhang S (2014) CT-Guided Core Needle Biopsy of Peripheral Lung Lesions with Onsite Adequate Evaluation: Review of 215 Cases. J Cytol Histol S4: 018. doi:10.4172/2157-7099.S4-018

Copyright: ( 92014 Liang L, et al. This is an open-access article distributed under the terms of the Creative Commons Attribution License, which permits unrestricted use, distribution, and reproduction in any medium, provided the original author and source are credited. 


\section{Materials and Methods}

Institutional Review Board approval for this study was obtained. The cytopathology archives of Memorial Hermann Hospital were retrospectively searched for CT-CN of peripheral lung lesions between January 1, 2009 and December 31, 2012. There were 215 cases during this four-year period. Chest wall, pleural or mediastinal biopsies were not included in this study. All the procedures were performed by radiologists. A pathologist was present during the procedure for rapid on-site cytology evaluation (ROSA). For preparing touch preparation cytology for the on-site evaluation, the cores were gently rolled against glass slides to obtain imprint smears. The slides were air-dried and stained with Diff-Quik for immediate on-site evaluation to determine the adequacy of the specimen. Additional slides of touch preparation were fixed in cytology preservative (CytoLyt) and later stained with Papanicolaou stain for further cytological assessment. The core biopsies were fixed in formalin and embedded in paraffin for routine $\mathrm{H} \& \mathrm{E}$ evaluation.

Information including age, lesion size, number of passes, immunohistochemistry, histochemical stains, onsite adequate assessment and final diagnosis was collected from the cytopathology reports. Clinical information including patients' history, imaging

\begin{tabular}{|l|c|}
\hline Diagnosis & Number of cases \\
\hline I. Benign & 70 \\
\hline Granulomatous inflammation & 10 \\
\hline Organizing pneumonia & 10 \\
\hline Fungal infection & 3 \\
\hline Acid fast bacilli infection & 1 \\
\hline Amyloidoma & 1 \\
\hline Lung elastosis & 1 \\
\hline Nonspecific & 44 \\
\hline II. Malignant & 132 \\
\hline Squamous cell carcinoma & 25 \\
\hline Adenocarcinoma & 40 \\
\hline NSCLS with both adeno and squamous components & 10 \\
\hline NSCLS, favor adenocarcinoma & 7 \\
\hline NSCLS, favor squamous & 3 \\
\hline NSCLS, NOS & 2 \\
\hline Small cell carcinoma or neuroendocrine & 11 \\
\hline Metastatic carcinoma & 26 \\
\hline Metastatic melanoma & 2 \\
\hline Metastatic sarcoma & 2 \\
\hline Malignant spindle cell neoplasm & 1 \\
\hline Mesothelioma & 1 \\
\hline B-cell lymphoma & 1 \\
\hline Thymoma & 13 \\
\hline III. Atypical & \\
\hline
\end{tabular}

Table 1: Classification of benign, malignant and atypical lesions. studies was collected from Electronic Medical Records (EMR). The follow-up surgical pathology diagnosis in EMR was reviewed.

Statistical analyses were performed using SPSS Software. Chisquare tests were used to compare lesions with different sizes and pass numbers. P-values less than 0.05 were considered statistically significant.

\section{Results}

There were a total of 215 specimens during the study period obtained from 207 patients (119 male and 88 female). The mean age of patients was 66 years ( 11 to 94 years). The average lesion size was 3.46 centimeters ( 0.4 to 20 centimeters) in 190 cases with available information. The average passes were 3.3 per lesion ( 1 to 10 passes per lesion).The average number of total slides submitted was 3.9 (1 to 19). The average number of Diff-Quick slides was 2.6 ( 1 to 10). The average number of Papanicolaou slides was 1.3 (0 to 9).

The final cytology diagnoses included 132 malignancies, 70 benign diagnoses, and 13 atypical diagnoses (Table 1). Twenty-four cases had follow-up surgical resections (11.2\%). There were two false negative diagnoses due to sampling errors (Table 2). There was no false positive diagnosis. The positive predictive value was $100 \%$ (16/16 cases). Five cases with diagnosis of "atypical" had follow-up surgical resections. One lesion was diagnosed as inflammatory pseudotumor, and the other four lesions were diagnosed as malignant neoplasm in the follow-up resection specimen, including three primary lung neoplasms and one metastatic carcinoma (Table 2).

Immunohistochemistry was performed in 137 cases, including 111 malignant, 11 atypical and 15 benign. The average number of immunostains performed per case was 5 ( 2 to 21$)$. The commonly used markers include CK7, CK20, TTF-1, p63, CK5/6, and Napsin A (Figure 1). Histochemical stains were performed in 53 cases. The most common histochemical stains were acid fast bacilli (AFB), and Grocott's Methenamine Silver (GMS) stain. Fungal infection was confirmed by GMS stain in three cases, including one coccidioidomycosis, one crytptococcus and one fungus not specified. One case of mycobacterial infection was confirmed by AFB stain (Figure 2). One case of pulmonary amyloidosis was confirmed by Congo red stain, and both touch preparation and CT-CN showed amorphous paucicellular material that stained orange with Congo red and exhibited apple-green birefringence under polarized light (Figure 3). Of note, electron microscopy was performed in two cases, and molecular tests were performed in 15 cases. Flow cytometry and FISH were performed in one case of B cell lymphoma.

In the 204 patients with available information of post-CT-CN chest X-ray, $140(68.6 \%)$ had no pneumothorax, and $64(31.4 \%)$ had pneumothorax including 48 mild (23.5\%), 3 moderate (1.5\%), 2 severe (1\%), 3 tension pneumothorax (1.5\%), and 8 with chest tubes placed during procedure (3.9\%). Two patients had pre-existing pneumothorax

\begin{tabular}{|c|c|c|c|c|c|}
\hline Case & Age & Lesion size (cm) & Core needle biopsy diagnosis & Follow-up procedure & Resection Diagnosis \\
\hline 1 & 65 & 1.4 & Negative for malignancy & Lobectomy & Squamous cell carcinoma \\
\hline 2 & 75 & 1.9 & Negative for malignancy & Wedge resection & Adenocarcinoma \\
\hline 3 & 73 & 5.3 & Atypical & Lobectomy & Inflammatory pseudotumor \\
\hline 4 & 80 & 1.0 & Atypical & Pneumectomy & Adenocarcinoma \\
\hline 5 & 80 & 2.3 & Atypical & Wedge resection & Adenosquamous carcinoma \\
\hline 6 & 63 & 1.1 & Atypical & Wedge resection & Metastatic hepatocellular carcinoma \\
\hline 7 & 68 & 2.5 & Atypical & Lobectomy & Adenocarcinoma \\
\hline
\end{tabular}

Table 2: Summary of false negative cases and cases that definite diagnosis cannot be made on core needle biopsy (only cases with follow-up resections included). 
Citation: Liang L, Liu J, Buryanek J, Zhang S (2014) CT-Guided Core Needle Biopsy of Peripheral Lung Lesions with Onsite Adequate Evaluation: Review of 215 Cases. J Cytol Histol S4: 018. doi:10.4172/2157-7099.S4-018

A

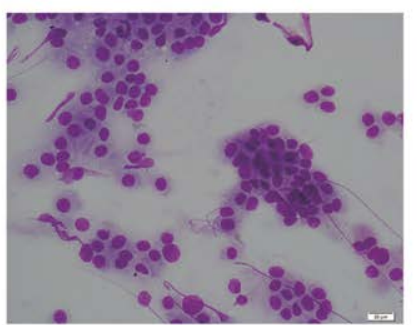

$\mathrm{C}$

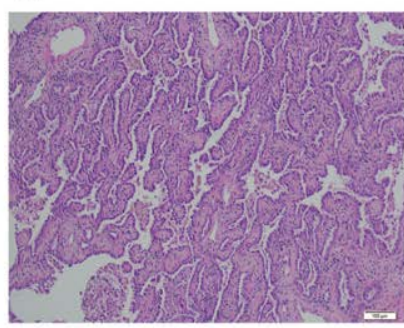

E

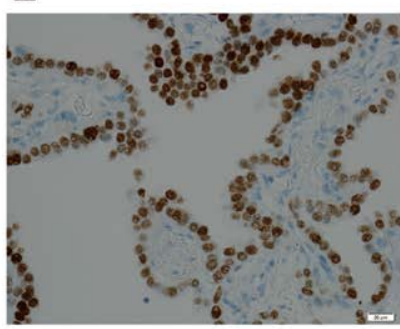

B

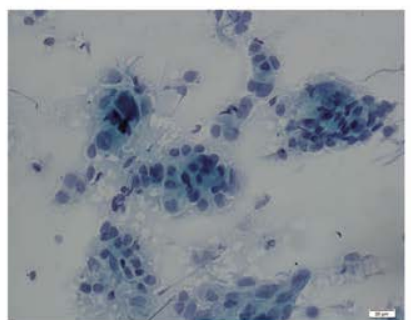

D

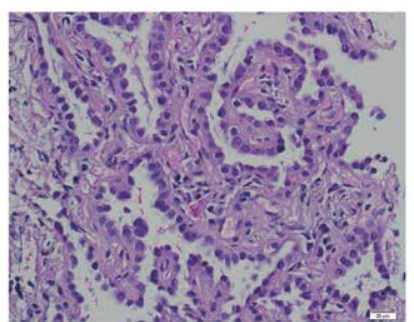

$\mathrm{F}$

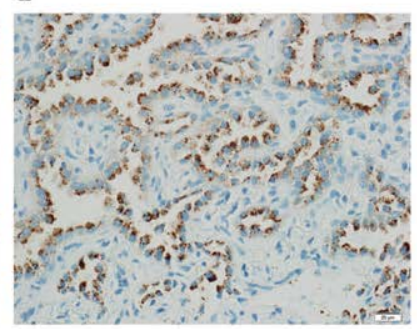

Figure 1: A case of lung adenocarcinoma had morphologic features of nonmucinous adenocarcinoma in situ (AIS). The Touch preparation slides showed cellular smears with some relative uniform tumor cells with moderate amount of cytoplasm without prominent nucleoli (A, Diff-Quik, 400x; B, Pap stain, 400x). The core tissue demonstrated the neoplastic cells with a bronchial alveolar growth pattern and relative bland nuclei, and there is no evidence of invasion (C, H\&E, 100x; D, H\&E, 400x). Tumor cells are positive for TTF1 (E, 400x) and Napsin A (F, 400x).

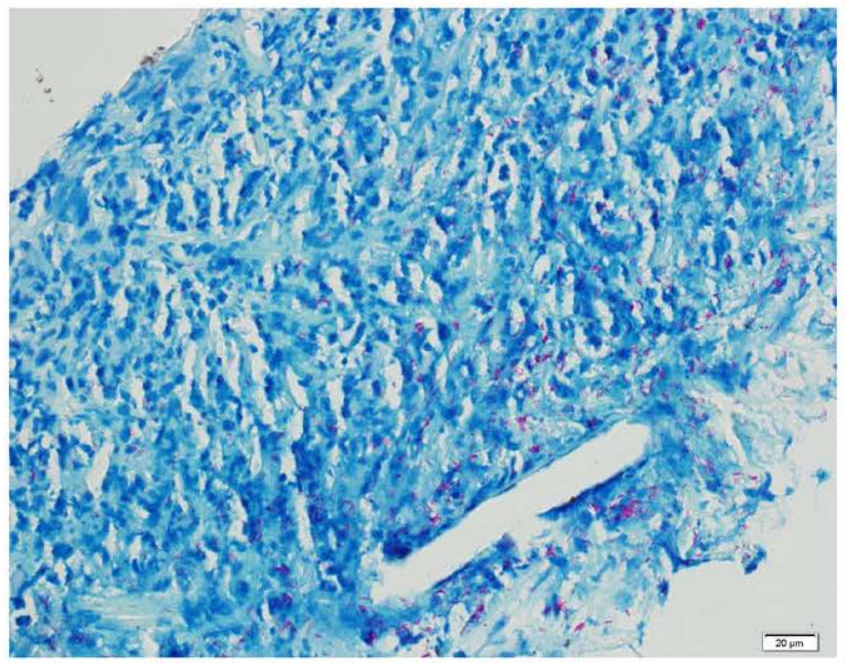

Figure 2: A case of lung mycobacterial infection showed many acid fast bacilli organisms with AFB staining (400x).

A

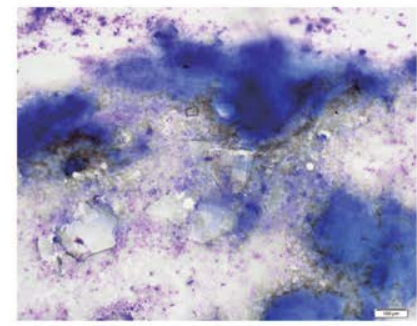

$\mathrm{C}$

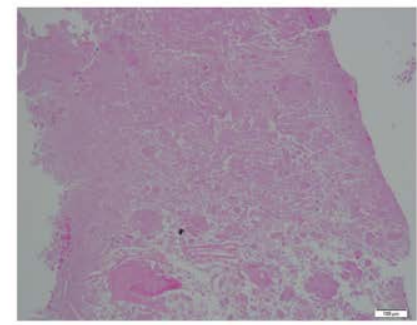

$\mathrm{D}$
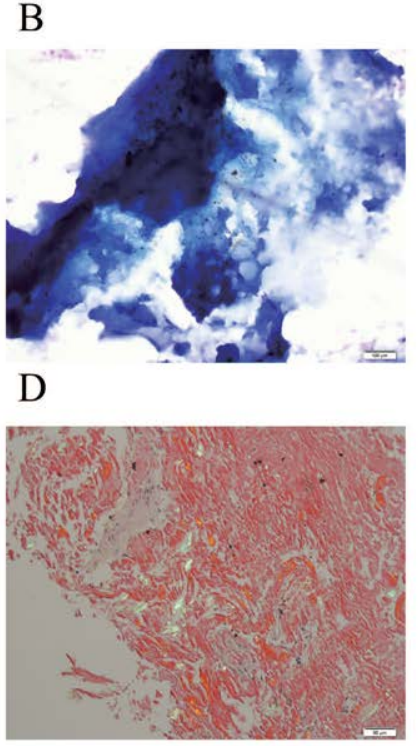

Figure 3: Acase of pulmonary amyloidoma showed abundant blue amorphorous material and some cholesterol crystals. (A-B, Diff-Quik, 100x), and core needle tissue showed abundant eosinophilic acellular material (C, H\&E, 100x), and Congo red stain showed classic "apple-green" birefringence of amyloid (D, Congo Red stain with polarization, 200x).

and chest tube placement before the procedure and weren't included here. Mild pneumothorax was defined as tiny apical or 10\%- $20 \%$ lung compression; moderate pneumothorax was defined as $30 \%-40 \%$ lung compression; severe pneumothorax was defined as $50 \%-60 \%$ lung compression; tension pneumothorax was diagnosed by the clinician and documented in the radiological reports or clinical notes. During the core needle biopsy procedures, the radiologists decided to insert a chest tube in 8 patients to prevent severe pneumothorax.

The cases were divided into three groups according to the lesion sizes $(<=2.0 \mathrm{~cm}, 2.1-5 \mathrm{~cm},>5.0 \mathrm{~cm})$. There was no statistically significant difference in pneumothorax rate among these three groups (Table 3, $\mathrm{p}>0.05)$. When the cases were divided into three groups according to the number of passes ( 1 to 2 passes, 3 to 4 passes, and 5 passes or more). There was no statistically significant difference in pneumothorax rate among these three groups (Table 4, $\mathrm{p}>0.05$ ).

In addition, molecular test results were available in 15 cases, including successful testing $(\mathrm{n}=11)$ and quantity not sufficient $(\mathrm{n}=4)$.

\begin{tabular}{|c|c|c|c|c|c|}
\hline \multirow{2}{*}{$\begin{array}{c}\text { Lesion size } \\
\text { (cm) }\end{array}$} & $\begin{array}{c}\text { Number of } \\
\text { cases }\end{array}$ & \multicolumn{4}{|c|}{ Pneumothorax } \\
\cline { 3 - 6 } & Negative & Positive & NA & Rates of pneumothorax \\
\hline$<=2.0$ & 69 & 42 & 24 & 3 & $36.4 \%(24 / 66)$ \\
\hline 2.1 to 5.0 & 83 & 53 & 26 & 4 & $32.9 \%(26 / 79)$ \\
\hline$>5.0$ & 38 & 26 & 9 & 3 & $25.7 \%(9 / 35)$ \\
\hline
\end{tabular}

Table 3: Comparison of core needle biopsies with different lesion sizes (in 190 cases).

\begin{tabular}{|c|c|c|c|c|c|}
\hline \multirow{2}{*}{$\begin{array}{c}\text { Number of } \\
\text { passes }\end{array}$} & $\begin{array}{c}\text { Number of } \\
\text { cases }\end{array}$ & \multicolumn{4}{|c|}{ Pneumothorax } \\
\cline { 3 - 6 } & Negative & Positive & NA & Rates of pneumothorax \\
\hline 1 or 2 & 79 & 53 & 22 & 4 & $29.3 \%(22 / 75)$ \\
\hline 3 or 4 & 94 & 60 & 28 & 6 & $31.8 \%(28 / 88)$ \\
\hline 5 or above & 42 & 27 & 14 & 1 & $34.1 \%(14 / 41)$ \\
\hline
\end{tabular}

Table 4: Comparison of core needle biopsies with different number of passes. 


\section{Discussion}

CT-CN with onsite cytology adequacy assessment using touch preparation has a low unsatisfactory rate $[2,9,12,13]$. In our study, the final adequate rate in our study was $99.5 \%$ with only one unsatisfactory case, and the satisfactory rates of the onsite evaluation were variable depending on the cytology diagnosis. The onsite evaluation satisfactory rates were $99.2 \%$ (131/132) for malignant diagnosis, $88.6 \%(62 / 70)$ for benign diagnosis, and $76.9 \%$ (10/13) for atypical diagnosis. A definite diagnosis (either positive or negative for malignancy) was made in $94 \%$ of the cases in our study and the atypical rate was $6 \%$. CT-CN with onsite cytology adequate assessment also provided more accurate classification of malignant tumors (Table 1). Furthermore, a specific diagnosis was made in $37 \%$ of cases in the benign category, including granulomatous inflammation, organizing pneumonia, fungal and mycobacterial infection, amyloidoma and lung elastosis.

CT-CN and surgical resection correlation was performed in 24 cases (11.2\%). There were two false negative diagnoses due to sampling errors (Table 2). There was no false positive diagnosis. In these two false negative cases, the malignant cells were not present in the touch preparation slides and the cores. So, a negative result of CT-CN does not totally exclude malignancy due to sampling error. Lesion size is a significant factor contributing to diagnostic accuracy in both FNA and biopsies [14-19]. Multiple studies reported higher rates and of false negative diagnosis of malignancy by FNA in relatively smaller lesions (equal or smaller than 1-3 cm). Mukherjee et al. [16] reported a negative predictive value of $66.67 \%$, and Montaudon et al. [19] reported a negative predictive value of $73.3 \%$. Kothary et al. [18] reported an accurate rate of $69.6 \%$. However, Gelbman et al. [14] reported that those with false negative results had significantly larger nodules compared with true negative cases (mean, $27 \mathrm{~mm}$ versus $17 \mathrm{~mm}, \mathrm{p}=0.04$ ). In our study, both lesions in the false negative cases were less than $2.0 \mathrm{~cm}$. Extensive necrosis can also lead to false negative in core needle biopsies, which wasn't identified in our two cases. It is crucial to correlate with clinical and radiological information and decide which patients need a follow-up resection. In addition, there were five patients with the core needle biopsy diagnosis in the "atypical" category underwent follow-up resections. The resection diagnosis were positive for malignant in four cases $(80 \%)$. Interestingly, the lesion sizes in all four cases were less than $3.0 \mathrm{~cm}$ (Table 2). This suggested that the patients in the "atypical" category needed to be followed up more carefully.

The core needle biopsy often has enough tissue for ancillary studies, including immunohistochemistry, histochemical stains, electron microscopy and molecular analysis, which makes it easier for tumor diagnosis and subclassification. In our study, immunohistochemistry was performed in 63\% (136/215) of the cases and histochemical study was performed in $24.7 \%$ (53/215) of the cases. In two cases, electron microscopy was performed and contributed to the diagnosis of mesothelioma and metastatic melanoma respectively. Molecular study was successfully performed in $73.3 \%$ (11/15) of the cases.

In the current study, we reported a pneumothorax rate of $31.4 \%$. Beslic et al. [2] compared the complication rate in CT- guided percutaneous trans-thoracic fine needle aspiration biopsies and core needle biopsies. They found that pneumothorax was detected in $9.7 \%$ of the patients with fine needle biopsy and $31.5 \%$ of the patients with the core needle biopsy respectively. However, other authors reported the pneumothorax rate was variable, ranging from less than $10 \%$ to more than $60 \%$ in fine needle aspiration and biopsies [19-25]. Wallace et al. [23] reported the highest rate of pneumothorax (62\%) in lung nodules
$1.0 \mathrm{~cm}$ or smaller underwent CT-guided transthoracic fine needle aspiration biopsy using either aspiration or capillary (nonaspiration) technique. Laspas et al. [24] reported a low pneumothorax rate (4\%) in fine needle aspiration (size of the lesions biopsied was from 0.6 to 10 $\mathrm{cm}$, mostly between 2 and $5 \mathrm{~cm}$ ). Poulou et al. [25] recently published a study of 1000 patients underwent fine needle biopsy, needle core biopsy or both, and found complication rate reached $9.7 \%$, including hemorrhage $(6.2 \%)$, pneumothorax $(2.8 \%)$, both hemorrhage and pneumothorax $(0.5 \%)$ and hemoptysis $(0.2 \%)$. Even though the incidence of pneumothorax is very low in their study, the incidence of hemorrhage is high, which was different from most other studies.

There are several other factors that can affect complication rate other than lesion size. Tachibana et al. [13] reported that core needle biopsy with immediate cytology evaluation can improve diagnostic performance and decrease the complication rate. Another possible cause of the big difference in complication rate is how the patients were monitored after the procedures. According to some authors, they observed the patients and post-procedure imaging studies were performed only when the patients developed symptoms, such as dyspnea, chest pain, or hemoptysis [24]. Some authors observed the inpatients in the ward, while the outpatients had CT scan or X-ray before they were dismissed from the hospital [24]. In our institution, post-procedure three-hour chest X-ray was routinely performed in all the patients, which identified more patients with mild pneumothorax. Since the clinical significant pneumothorax may take time to develop, we believe it may be beneficial for the patients to undergo routine post-procedure three-hour chest X-ray. In our study, the overall pneumothorax rate is about $31.4 \%$, but the majority of these patients had mild pneumothorax (23.5\%). The high rate in our study may be related to the close follow-up and the mandatory chest X-ray few hours after the procedure. In $3.9 \%$ of the patients, the radiologists decided to insert chest tubes during the procedure to present a significant pneumothorax from developing. It depended on the radiologists' experience which patient was treated with chest tube placement. Even though we observe a possible "trend" that the pneumothorax rates slightly increase with more number of passes, the difference was not statistically significant. Rizzo et al. [26] reported small lesions were significantly associated with the onset of parenchymal hemorrhage and pneumothorax. On the contrary, Kotharya et al. [18] studied 139 patients underwent CT-guided percutaneous fine-needle aspiration biopsy or 20-gauge core biopsy using an automated biopsy gun and found no correlation between nodule size and the incidence of complications. In our study, there was no significant difference in pneumothorax rate within three groups with different lesion sizes.

In 2011, a multidisciplinary expert panel representing the International Association for the Study of Lung Cancer (IASLC), the American Thoracic Society (ATS), and the European Respiratory Society (ERS) proposed a major revision of lung classification [11]. Since we retrospectively studied cases from 2009 and 2012, the majority of the cases were signed out before the new classification was implemented. In the future, it will be useful to further study the diagnosis and subclassification of lung cancers using the 2011 IASLC/ ATS/ERS Classification. Also recently, National Lung Screening Trial (NLST), a randomized trial funded by National Cancer Institute (NCI) published its available data and showed that lung cancer death rates can be reduced by $20 \%$ when a high-risk group is screened with a lowdose computer tomography (CT) scan compared to chest radiography [27]. It is still too early to tell if low-dose computer tomography (CT) should be used to screen the high risk patients in the general population without carefully considering the cost-effectiveness and possibility of 
Citation: Liang L, Liu J, Buryanek J, Zhang S (2014) CT-Guided Core Needle Biopsy of Peripheral Lung Lesions with Onsite Adequate Evaluation: Review of 215 Cases. J Cytol Histol S4: 018. doi:10.4172/2157-7099.S4-018

overdiagnosis [28]. However, it is possible that, in the near future, more lung cancers will be diagnosed at an early stage and more core needle biopsies will be performed in small lesions.

In summary, CT-CN with onsite cytology evaluation of touch preparation is a valuable tool in the initial diagnosis of peripheral lung lesions and it provides more accurate classification of malignant tumors and more specific diagnosis on non-neoplastic lung lesions.

\section{Acknowledgment}

The data has in part been presented at the Annual Meeting of College of American Pathologist (poster 109), Orlando, Florida, October 13-16, 2013.

\section{References}

1. Siegel R, Naishadham D, Jemal A (2013) Cancer statistics, 2013. CA Cancer J Clin 63: 11.

2. Beslic S, Zukic F, Milisic S (2012) Percutaneous transthoracic CT guided biopsies of lung lesions; fine needle aspiration biopsy versus core biopsy. Radiol Oncol 46: 19-22.

3. Lima CD, Nunes RA, Saito EH, Higa C, Cardona ZJ, et al. (2011) Results and complications of CT-guided transthoracic fine-needle aspiration biopsy of pulmonary lesions. J Bras Pneumol 37: 209-216.

4. Li H, Boiselle PM, Shepard JO, Trotman-Dickenson B, McLoud TC (1996) Diagnostic accuracy and safety of CT-guided percutaneous needle aspiration biopsy of the lung: comparison of small and large pulmonary nodules. AJR Am J Roentgenol 167: 105-109.

5. Geraghty PR, Kee ST, McFarlane G, Razavi MK, Sze DY, et al. (2003) CTguided transthoracic needle aspiration biopsy of pulmonary nodules: needle size and pneumothorax rate. Radiology 229: 475-481.

6. Cox JE, Chiles C, McManus CM, Aquino SL, Choplin RH (1999) Transthoracic needle aspiration biopsy: variables that affect risk of pneumothorax. Radiology 212: 165-168.

7. Yeow KM, Su IH, Pan KT, Tsay PK, Lui KW, et al. (2004) Risk factors of pneumothorax and bleeding: multivariate analysis of $660 \mathrm{CT}$-guided coaxial cutting needle lung biopsies. Chest 126: 748-754.

8. Johnson DH, Fehrenbacher L, Novotny WF, Herbst RS, Nemunaitis JJ, et al. (2004) Randomized phase II trial comparing bevacizumab plus carboplatin and paclitaxel with carboplatin and paclitaxel alone in previously untreated locally advanced or metastatic non-small-cell lung cancer. J Clin Oncol 22: 2184-2191.

9. Solomon SB, Zakowski MF, Pao W, Thornton RH, Ladanyi M, et al. (2010) Core needle lung biopsy specimens: adequacy for EGFR and KRAS mutational analysis. AJR Am J Roentgenol 194: 266-269.

10. Zhuang YP, Wang HY, Shi MQ, Zhang J, Feng Y (2011) Use of CT-guided fine needle aspiration biopsy in epidermal growth factor receptor mutation analysis in patients with advanced lung cancer. Acta Radiol 52: 1083-1087.

11. Travis WD, Brambilla E, Noguchi M, Nicholson AG, Geisinger KR, et al. (2011) International association for the study of lung cancer/american thoracic society/ european respiratory society international multidisciplinary classification of lung adenocarcinoma. J Thorac Oncol 6: 244-285.

12. Uskül BT, Türker H, Gökçe M, Kant A, Arslan S, et al. (2009) CT-guided transthoracic fine needle aspiration of pulmonary lesions: accuracy and complications in 134 cases. Tuberk Toraks 57: 177-185.

13. Tachibana K, Nakazato Y, Tsuchida S, Kazama T, Minato K, et al. (2013) Immediate cytology improves accuracy and decreases complication rate in real-time computed tomography-guided needle lung biopsy. Diagn Cytopathol 41: 1063-1068.
14. Gelbman BD, Cham MD, Kim W, Libby DM, Smith JP, et al. (2012) Radiographic and clinical characterization of false negative results from CT-guided needle biopsies of lung nodules. J Thorac Oncol 7: 815-820.

15. Minot DM, Jaben E, Aubry MC, Voss JS, Vine RL, et al. (2012) Evolution of transthoracic fine needle aspiration and core needle biopsy practice: A comparison of two time periods, 1996-1998 and 2003-2005. Diagn Cytopathol 40: 876-881.

16. Mukherjee S, Bandyopadhyay G, Bhattacharya A, Ghosh R, Barui G, et al. (2010) Computed tomography-guided fine needle aspiration cytology of solitary pulmonary nodules suspected to be bronchogenic carcinoma: Experience of a general hospital. J Cytol 27: 8-11.

17. Guimarães MD, Chojniak R, Gross JL, Bitencourt AGV (2009) Predictive success factors for CT-guided fine needle aspiration biopsy of pulmonary lesions. Clinics (Sao Paulo) 64: 1139-1144.

18. Kothary N, Lock L, Sze DY, Hofmann LV (2009) Computed tomography-guided percutaneous needle biopsy of pulmonary nodules: impact of nodule size on diagnostic accuracy. Clin Lung Cancer 10: 360-363.

19. Montaudon M, Latrabe V, Pariente A, Corneloup O, Begueret $\mathrm{H}$, et al. (2004) Factors influencing accuracy of CT-guided percutaneous biopsies of pulmonary lesions. Eur Radiol 14: 1234-1240.

20. Guimarães MD, Andrade MQ, Fonte AC, Benevides G, Chojniak R, et al. (2010) Predictive complication factors for CT-guided fine needle aspiration biopsy of pulmonary lesions. Clinics (Sao Paulo) 65: 847-850.

21. Heck SL, Blom P, Berstad A (2006) Accuracy and complications in computed tomography fluoroscopy-guided needle biopsies of lung masses. Eur Radiol 16: $1387-1392$.

22. Wu CC, Maher MM, Shepard JA (2011) Complications of CT-guided percutaneous needle biopsy of the chest: prevention and management. AJR Am J Roentgenol 196: W678-682.

23. Wallace MJ, Krishnamurthy S, Broemeling LD, Gupta S, Ahrar K, et al. (2002) CT-guided percutaneous fine-needle aspiration biopsy of small $(<$ or $=1-\mathrm{cm})$ pulmonary lesions. Radiology 225: 823-828.

24. Laspas F, Roussakis A, Efthimiadou R, Papaioannou D, Papadopoulos S, et al. (2008) Percutaneous CT-guided fine-needle aspiration of pulmonary lesions: Results and complications in 409 patients. J Med Imaging Radiat Oncol 52: 458-462.

25. Poulou LS, Tsagouli P, Ziakas PD, Politi D, Trigidou R, et al. (2013) Computed tomography-guided needle aspiration and biopsy of pulmonary lesions: a single-center experience in 1000 patients. Acta Radiol 54: 640-645.

26. Rizzo S, Preda L, Raimondi S, Meroni S, Belmonte M, et al. (2011) Risk factors for complications of CT-guided lung biopsies. Radiol Med 116: 548-563.

27. National Lung Screening Trial Research Team, Aberle DR, Adams AM, Berg CD, Black WC, et al. (2011) Reduced lung-cancer mortality with low-dose computed tomographic screening. N Engl J Med 365: 395-409.

28. Heuvers ME, Wisnivesky J, Stricker BH, Aerts JG (2012) Generalizability of results from the National Lung Screening Trial. Eur J Epidemiol 27: 669-672.
This article was originally published in a special issue, Histology and Histopathology handled by Editor(s). Borislav A. Alexiev, University of Maryland Medical Center, USA 